\title{
Successful management of shortening of overlapping segment and subsequent restenosis at the gap between two second-generation stents in the left anterior descending artery
}

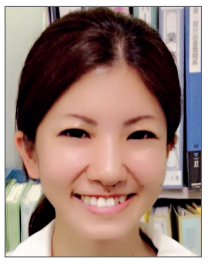

Nozomi Kotoku ${ }^{1}, \mathrm{MD}, \mathrm{PhD}$; Yuki Ishibashi ${ }^{1 *}, \mathrm{MD}, \mathrm{PhD}$; Yasuhiro Tanabe ${ }^{1}, \mathrm{MD}, \mathrm{PhD}$;

Akiyuki Kotoku², MD, PhD; Hiroshi Yamazaki ${ }^{1}$, MD; Shingo Kuwata ${ }^{1}, \mathrm{MD}$, PhD;

Takanobu Mitarai ${ }^{1}$, MD; Kazuaki Okuyama ${ }^{1}, \mathrm{MD}, \mathrm{PhD}$; Kihei Yoneyama ${ }^{1}, \mathrm{MD}, \mathrm{PhD}$;

Takumi Higuma ${ }^{1}, \mathrm{MD}, \mathrm{PhD}$; Tomoo Harada ${ }^{1}, \mathrm{MD}, \mathrm{PhD}$; Yoshihiro J. Akashi ${ }^{1}$, MD, PhD

1. Division of Cardiology, Department of Internal Medicine. St. Marianna University School of Medicine, Kawasaki, Kanagawa, Japan; 2. Division of Radiology, St. Marianna University School of Medicine, Kawasaki, Kanagawa, Japan

This paper also includes supplementary data published online at: www.asiaintervention.org

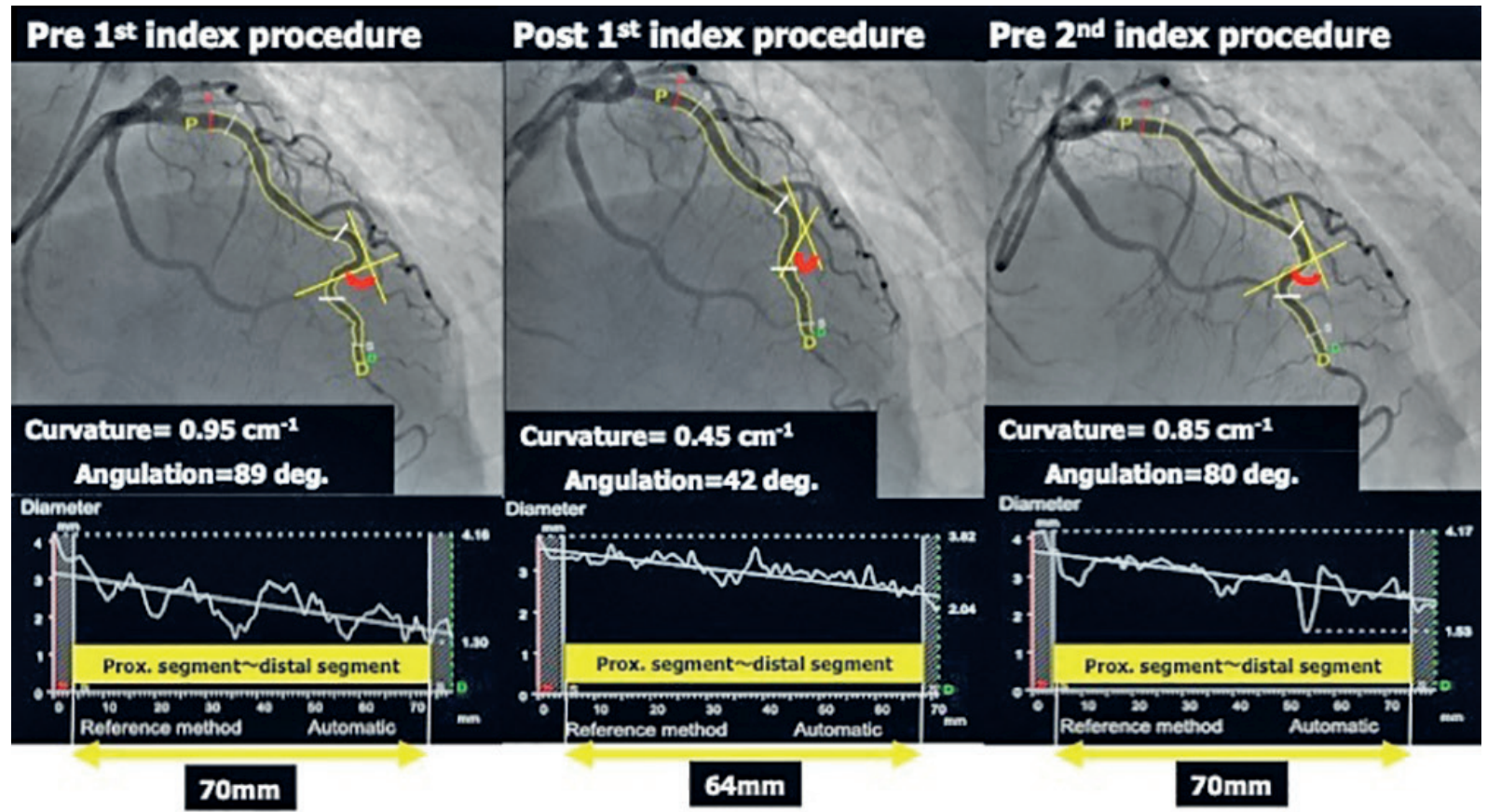


A 74-year-old man with silent myocardial ischaemia underwent percutaneous coronary intervention to overlap a $3.0 \times 38 \mathrm{~mm}$ everolimus-eluting stent (EES; XIENCE Alpine ${ }^{\mathrm{TM}}$, Abbott Vascular, Santa Clara, CA, USA) and a $2.5 \times 16 \mathrm{~mm}$ everolimus-eluting platinum chromium stent (PtCr-EES; Promus PREMIERTM, Boston Scientific Corp., Marlborough, MA, USA) in the mid-portion of the left anterior descending artery (LAD) (Moving image 1). Nine months after the stent implantation, the patient was readmitted to our hospital due to exertional chest pain. Coronary angiography (CAG) revealed significant restenosis at the mid-portion of the tortuous LAD segment (Moving image 2). Coronary computed tomography showed a distal stent fracture and shortening of the overlapping segment (Moving image 3). Optical coherence tomography (OCT) was also performed for the detailed examination. No significant luminal narrowing of the fractured segment was found (OCT, $3.97 \mathrm{~mm}^{2}$ ); whereas the minimum lumen area of the gaps between the two stented segments was $1.07 \mathrm{~mm}^{2}$ (OCT) (Moving image 4). A $3.0 \times 12 \mathrm{~mm}$ PtCr-EES was successfully overlapped at the gap segment, from the proximal to the previously overlapped segment (Moving image 5). Follow-up CAG at 9 months after the second procedure resulted in no clinical events.

In the present case, the quantitative $\mathrm{CAG}$ analysis suggested that shortening of the overlapping segment and the gap between the two second-generation DESs might have occurred due to 1) straightening of the vessel curves after metallic stent implantation and 2) geometric changes caused by stent placement (Figure).

After the gap, caused by continuous stress during cardiac cycle, was created between the two second-generation DESs in the LAD, mechanical stress imposed on the stent edges would cause vessel wall injury and inflammation, characterised by the deposition of platelets and fibrin, as well as adhesions of circulating neutrophils and macrophages, which might lead to restenosis.

Previous studies reported that the PtCr-EESs with higher bending flexibility had greater fracture resistance against more severe angulation ranges than the other second-generation drug-eluting stents. In this case, the PtCr-EES was thus selected for significant restenosis at the gap between the two metal stents.

\section{Conflict of interest statement}

The authors have no conflicts of interest to declare.

\section{Supplementary data}

Moving image 1. CAG after percutaneous coronary intervention and stenting of the LAD.

Moving image 2. CAG showing significant restenosis at the midportion of the tortuous LAD segment.

Moving image 3. Coronary computed tomography showing the distal stent fracture and shortening of overlapping segment.

Moving image 4. OCT showing the distal stent fracture and the gaps between the two stented segments.

Moving image 5. CAG after a PtCr-EES successfully overlapped the gap segment.

The supplementary data are published online at: www.asiaintervention.org 